hep-ph/9806286

June 1998

\title{
Unitarity and the scale of fermion mass generation
}

\author{
S. Jäger淌 and S. Willenbrock尚 \\ Department of Physics \\ University of Illinois \\ 1110 West Green Street \\ Urbana, IL 61801
}

\begin{abstract}
We study the upper bound on the scale of fermion mass generation in a two-Higgsdoublet model. If the model is weakly-coupled, the scale of fermion mass generation is much less than the Appelquist-Chanowitz unitarity bound. However, if we allow some dimensionless Higgs self-couplings to become large, the Appelquist-Chanowitz unitarity bound can be saturated. The unitarity bound on the scale of top-quark mass generation is about $3 \mathrm{TeV}$, which may be within the reach of future colliders.
\end{abstract}

\footnotetext{
*Address after Nov. 1998: Physik-Department, Technische Universität München, James-Franck-Strasse, 85748 Garching, Germany.

${ }^{\dagger}$ Email: sjaeger@physik.tu-muenchen.de

$\ddagger$ Email: willen@uiuc.edu
} 
Many years ago, Appelquist and Chanowitz [1] derived an upper bound on the scale of fermion mass generation for a fermion of mass $m_{f}$ :円

$$
\Lambda_{f}<\frac{8 \pi v^{2}}{\sqrt{3 N_{c}} m_{f}}
$$

where $v^{2} \equiv\left(\sqrt{2} G_{F}\right)^{-1}$ and $N_{c}=3$ for quarks and 1 for leptons. This bound was obtained by considering the scattering process $f \bar{f} \rightarrow V_{L} V_{L}[V=W, Z ; L$ denotes longitudinal polarization (helicity zero)] in the absence of a Higgs boson. The resulting amplitude grows linearly with the center-of-mass energy, and violates the condition of tree-level unitarity at the energy $\Lambda_{f}$ given in Eq. (11).

If this bound is relevant, it has interesting implications for future high-energy colliders. The numerical value of the bound for the top quark, with a mass of $173.9 \pm 5.2 \mathrm{GeV}$ [3], is approximately $3 \mathrm{TeV}$. This energy could serve as a benchmark for the study of top-quark mass generation via $V_{L} V_{L} \rightarrow t \bar{t}$ at future hadron [4, 5, 6, 7]. [2 $e^{+} e^{-}$[6, 13, 14, 7, 15], and $\mu^{+} \mu^{-}$[16] colliders, in much the same way that

$$
\Lambda_{E W S B}^{2}=8 \pi v^{2} \approx(1.2 \mathrm{TeV})^{2}
$$

(obtained from the consideration of $V_{L} V_{L} \rightarrow V_{L} V_{L}$ in the absence of a Higgs boson [17, 2]) serves as the benchmark for electroweak symmetry breaking (EWSB).

The relevance of the bound in Eq. (1) was already questioned in the original paper of Appelquist and Chanowitz. In the standard Higgs model, the Higgs boson is responsible for fermion mass generation, yet its mass cannot exceed approximately $800 \mathrm{GeV}$ [18, , 3 well below the bound in Eq. (1) for all known fermions. In extended Technicolor (ETC) models [19, 20], the scale of fermion mass generation is the mass of the ETC gauge boson

$$
M_{E T C} \sim g_{E T C}\left(\frac{v^{3}}{m_{f}}\right)^{1 / 2}
$$

which scales with $m_{f}$ differently from Eq. (1), and is much less than $\Lambda_{f}$ for all known fermions. Furthermore, the process $f \bar{f} \rightarrow V_{L} V_{L}$ ceases to grow with energy at an energy of order $\Lambda_{E W S B}$ rather than $M_{E T C}$ [1]. Several years later, Golden [21] revisited the issue of the relevance of the bound in models with a Higgs boson, and found a bound which scales with $m_{f}$ like Eq. (3) rather than Eq. (11). Thus the relevance of the bound in Eq. (四) remains an open and important question.

In this paper we study the scale of fermion mass generation in the context of a two-Higgsdoublet model. We imagine that the Higgs scalar responsible for unitarizing $V_{L} V_{L} \rightarrow V_{L} V_{L}$ is "light" fl and we derive an upper bound on the mass of the heavy Higgs scalar which is

\footnotetext{
${ }^{1}$ The strictest bound is obtained by considering the spin-singlet, weak-isosinglet, color-singlet amplitude [2].

${ }^{2}$ At a hadron collider, one can also probe $t \bar{t} \rightarrow V_{L} V_{L}$, either at tree level through $g g \rightarrow t \bar{t} V_{L} V_{L}$ [8] or at one loop through $g g \rightarrow V_{L} V_{L}$ via a top-quark loop [9, 10, 11, 12].

${ }^{3}$ This upper bound is obtained by demanding that the Higgs mass be less than the intrinsic cutoff of the theory 23.

${ }^{4}$ A "light" Higgs boson is one whose mass is less than about $800 \mathrm{GeV}$.
} 
responsible for unitarizing $f \bar{f} \rightarrow V_{L} V_{L}$. We find that if all dimensionless Higgs self-couplings are kept fixed, the bound on the mass of the heavy Higgs scalar scales with $m_{f}$ like Eq. (3). However, if some of the dimensionless Higgs self-couplings are allowed to grow with the mass of the heavy Higgs scalar, the bound on the mass of this Higgs scalar is instead given by the Appelquist-Chanowitz bound, Eq. (11).

Before we begin our analysis, let us recall the meaning of unitarity bounds in effective field theories [1, 22]. In the absence of a Higgs boson, a perturbative calculation of the scattering amplitude for $V_{L} V_{L} \rightarrow V_{L} V_{L}$ yields an expansion in powers of $E^{2} / v^{2}$. This expansion becomes useless once the expansion parameter is of order unity. One can use unitarity to estimate this energy [17, 2], which yields $E \sim \Lambda_{E W S B}$ (Eq. (2)). The addition of a "light" Higgs boson to the theory changes the expansion parameter to $m_{h}^{2} / v^{2}$ (for $E^{2}>>m_{h}^{2}$ ); one says that the Higgs boson "unitarizes" the theory. The largest Higgs mass allowed is approximately 800 $\mathrm{GeV}$ [18], which nearly saturates the bound $\Lambda_{E W S B}$.

Now add a massive fermion of mass $m_{f}$ to the theory, again in the absence of a Higgs boson. In processes involving the massive fermion, one finds an additional expansion parameter, $m_{f} E / v^{2}$, along with the expansion parameter $E^{2} / v^{2}$. Again, unitarity can be used to estimate the energy at which this additional expansion parameter is of order unity, and the result is $E \sim \Lambda_{f}$ (Eq. (1D)). This interpretation of the Appelquist-Chanowitz bound is supported by the analysis of one-loop diagrams in section 2 of Golden [21].

Whatever physics unitarizes $V_{L} V_{L} \rightarrow V_{L} V_{L}$ need not necessarily unitarize $f \bar{f} \rightarrow V_{L} V_{L}$. In the standard Higgs model, the same Higgs boson is responsible for unitarizing both processes. We therefore consider a two-Higgs-doublet model, with only one Higgs field coupled to a given fermion. This can be arranged by imposing a discrete symmetry $\Phi_{1} \rightarrow-\Phi_{1}$ on one of the Higgs fields, such that only the Higgs field $\Phi_{2}$ has Yukawa couplings to a given fermion [24]. The most general CP-symmetric scalar potential, with the discrete symmetry $\Phi_{1} \rightarrow-\Phi_{1}$ softly broken, is [25, 26, 27]

$$
\begin{aligned}
V\left(\Phi_{1}, \Phi_{2}\right) & =m_{11}^{2} \Phi_{1}^{\dagger} \Phi_{1}+m_{22}^{2} \Phi_{2}^{\dagger} \Phi_{2}-m_{12}^{2}\left[\Phi_{1}^{\dagger} \Phi_{2}+\Phi_{2}^{\dagger} \Phi_{1}\right] \\
& +\frac{1}{2} \lambda_{1}\left(\Phi_{1}^{\dagger} \Phi_{1}\right)^{2}+\frac{1}{2} \lambda_{2}\left(\Phi_{2}^{\dagger} \Phi_{2}\right)^{2}+\lambda_{3}\left(\Phi_{1}^{\dagger} \Phi_{1}\right)\left(\Phi_{2}^{\dagger} \Phi_{2}\right)+\lambda_{4}\left(\Phi_{1}^{\dagger} \Phi_{2}\right)\left(\Phi_{2}^{\dagger} \Phi_{1}\right) \\
& +\frac{1}{2} \lambda_{5}\left[\left(\Phi_{1}^{\dagger} \Phi_{2}\right)^{2}+\left(\Phi_{2}^{\dagger} \Phi_{1}\right)^{2}\right]
\end{aligned}
$$

where all parameters are real. The particle content of this model is two neutral Higgs scalars, $h^{0}$ and $H^{0}$; a neutral Higgs pseudoscalar, $A^{0}$; and a charged Higgs scalar, $H^{ \pm}$. Only the neutral Higgs scalars, $h^{0}$ and $H^{0}$, will be relevant for the ensuing discussion.

Our analysis of the model closely follows that of Haber [27]. The two Higgs fields acquire vacuum-expectation values $v_{1}$ and $v_{2}$, where $v_{1}^{2}+v_{2}^{2}=v^{2}$, and we define $\tan \beta \equiv v_{2} / v_{1}$, as usual. The neutral-Higgs-scalar mass eigenstates are related to the weak eigenstates by a rotation through the angle $\alpha$. Their masses are given by

$$
m_{H^{0}, h^{0}}^{2}=\frac{1}{2}\left(m_{S}^{2} \pm \sqrt{m_{S}^{4}-4 m_{A^{0}}^{2} m_{L}^{2}-4 m_{D}^{4}}\right)
$$

\footnotetext{
${ }^{5}$ Alternatively, one can couple a given fermion $f$ to the Higgs field $\Phi_{1}$ by letting $f_{R} \rightarrow-f_{R}$ under the discrete symmetry.
} 


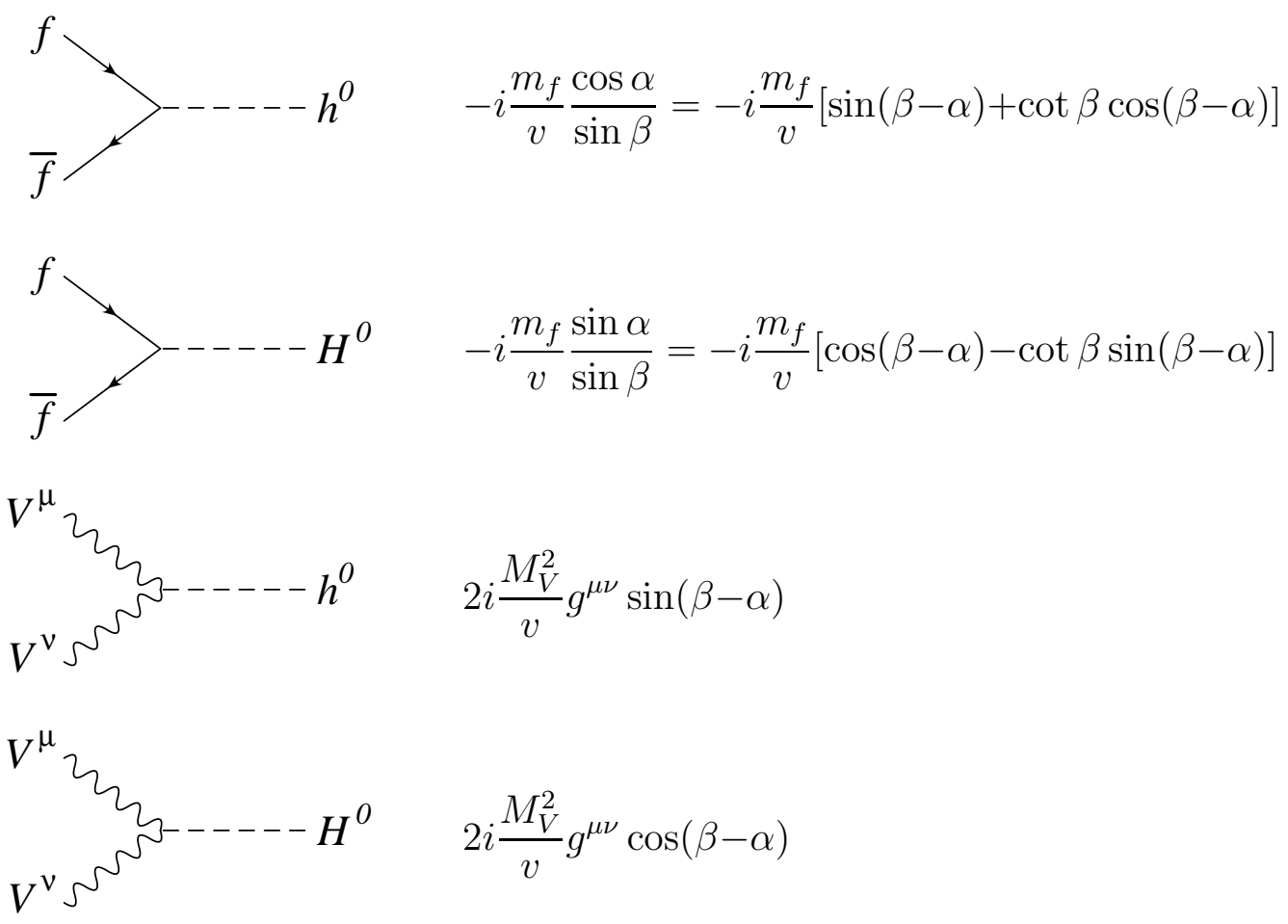

Figure 1: Couplings of the neutral Higgs scalars of a two-Higgs-doublet model to fermionantifermion and weak-vector-boson pairs.

where

$$
\begin{aligned}
m_{A^{0}}^{2} & =\frac{m_{12}^{2}}{s_{\beta} c_{\beta}}-v^{2} \lambda_{5} \\
m_{L}^{2} & \equiv v^{2}\left[\lambda_{1} c_{\beta}^{4}+\lambda_{2} s_{\beta}^{4}+2\left(\lambda_{3}+\lambda_{4}+\lambda_{5}\right) s_{\beta}^{2} c_{\beta}^{2}\right] \\
m_{D}^{2} & \equiv v^{2}\left[\lambda_{5}\left(\lambda_{1} c_{\beta}^{4}+\lambda_{2} s_{\beta}^{4}\right)+\left(\lambda_{1} \lambda_{2}+\lambda_{5}^{2}-\left(\lambda_{3}+\lambda_{4}\right)^{2}\right) s_{\beta}^{2} c_{\beta}^{2}\right]^{1 / 2} \\
m_{T}^{2} & \equiv v^{2}\left(\lambda_{1} c_{\beta}^{2}+\lambda_{2} s_{\beta}^{2}+\lambda_{5}\right) \\
m_{S}^{2} & \equiv m_{A^{0}}^{2}+m_{T}^{2}
\end{aligned}
$$

and where we have adopted the short-hand notation $s_{\beta} \equiv \sin \beta, c_{\beta} \equiv \cos \beta$.

The relevant Feynman rules for the Higgs scalars are listed in Fig. 1. Since we want the light Higgs scalar $h^{0}$ to (almost completely) unitarize $V_{L} V_{L} \rightarrow V_{L} V_{L}$, we want its coupling to vector bosons to be close to the standard-model value. Thus we are interested in the limit $\cos (\beta-\alpha)<<1$, where [27]

$$
\cos ^{2}(\beta-\alpha)=\frac{m_{L}^{2}-m_{h^{0}}^{2}}{m_{H^{0}}^{2}-m_{h^{0}}^{2}} .
$$

We show in Fig. 2 the Feynman diagrams which, taken together, unitarize $f \bar{f} \rightarrow V_{L} V_{L}$. The diagrams have a value equal to the same diagram in the standard Higgs model, times the factor shown to the right of each diagram. Since we are interested in the limit $\cos (\beta-$ 


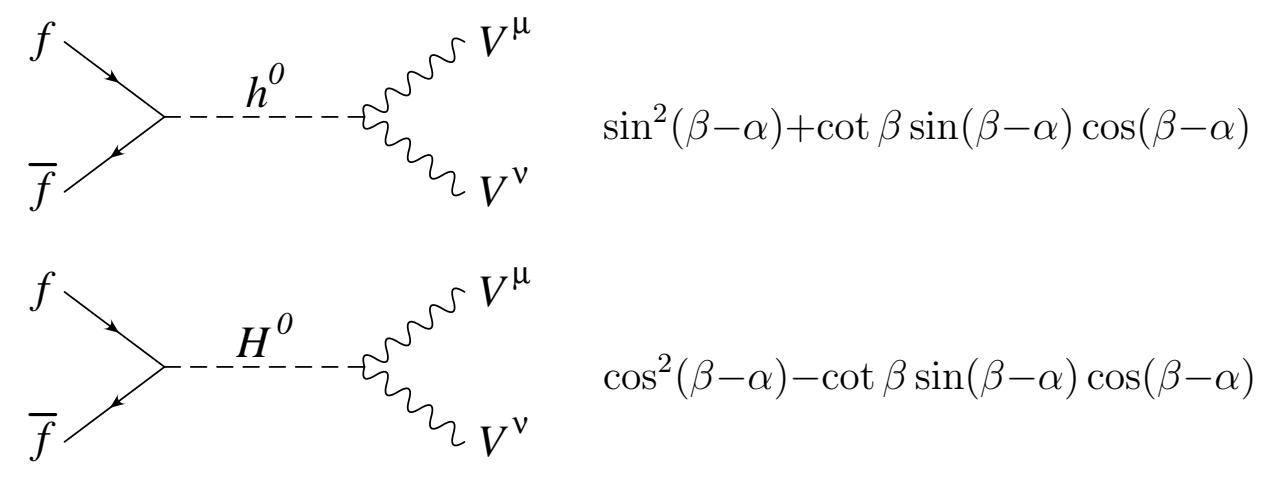

Figure 2: Diagrams which unitarize $f \bar{f} \rightarrow V_{L} V_{L}$ in a two-Higgs-doublet model.

$\alpha)<<1$, the only way that the diagram involving the heavy Higgs scalar $H^{0}$ can contribute significantly to the unitarization of $f \bar{f} \rightarrow V_{L} V_{L}$ is if

$$
\cot \beta \sin (\beta-\alpha) \cos (\beta-\alpha) \sim \mathcal{O}(1)
$$

or equivalently, $\operatorname{since} \sin (\beta-\alpha) \approx 1$,

$$
\cot \beta \sim 1 / \cos (\beta-\alpha)>>1 \text {. }
$$

This corresponds to a strong Yukawa coupling of the heavy Higgs scalar to the fermion (see Fig. 1), proportional to $\left(m_{f} / v\right) \cot \beta$.

To proceed further, we must introduce an additional piece of physics. Recall that the upper bound on the Higgs mass arises from the condition that it be less than the cutoff of the theory [23]. This condition turns out to be equivalent to the condition that the (running) Higgs self-interaction remain perturbative below the cutoff [18]. Similarly, the Yukawa coupling of a fermion must remain perturbative below the cutoff [28, 29]. This puts an upper bound on the Yukawa coupling, which can be estimated from the unitarity of $f \bar{f} \rightarrow f \bar{f}$ 30, 2]. One finds

$$
\frac{m_{f}}{v} \cot \beta<\left(\frac{4 \pi}{N_{c}}\right)^{1 / 2} .
$$

Combining Eqs. (9) and (10) gives

$$
\cos (\beta-\alpha)>\left(\frac{N_{c}}{4 \pi}\right)^{1 / 2} \frac{m_{f}}{v} .
$$

This is a constraint on how small $\cos (\beta-\alpha)$ can be, and still have the heavy Higgs scalar $H^{0}$ contribute significantly to the unitarization of $f \bar{f} \rightarrow V_{L} V_{L}$.

To convert Eq. (11) into an upper bound on the mass of the heavy Higgs scalar $H^{0}$, we must consider how $\cos (\beta-\alpha)$ scales with $m_{H^{0}}$. If we hold the dimensionless Higgs self-couplings $\lambda_{i}$ fixed, and increase the mass of the heavy Higgs scalar by increasing the parameter $m_{12}$, Haber [27] has shown that

$$
\cos ^{2}(\beta-\alpha) \sim\left(\frac{\lambda_{i} v^{2}}{m_{H^{0}}^{2}}\right)^{2} .
$$

\footnotetext{
${ }^{6}$ This follows from Eq. (7), due to a cancellation between the two terms in the numerator for large $m_{S}^{2}$.
} 
Combining Eqs. (11) and (12) yields

$$
m_{H^{0}}<\left(\frac{4 \pi}{N_{c}}\right)^{1 / 4}\left(\frac{\lambda_{i} v^{3}}{m_{f}}\right)^{1 / 2}
$$

which scales with $m_{f}$ like Eq. (3). This is an example of a model in which the scale of fermion mass generation scales with $m_{f}$ as in extended Technicolor, yet the process $f \bar{f} \rightarrow V_{L} V_{L}$ is unitarized at the scale of fermion mass generation, $m_{H^{0}}$, rather than at $\Lambda_{E W S B}$, Eq. (2)). The same scaling, but via a different mechanism, is found in section 3 of Golden [21] from an analysis of higher-dimension operators; the model we are considering only involves operators of dimension four or less. For $\lambda_{i} \approx 4 \pi$ (the largest value of the coupling such that it is perturbative), Eq. (13) yields an upper bound of about $1.5 \mathrm{TeV}$ on the scale of top-quark mass generation.

If we instead allow some of the $\lambda_{i}$ to increase with the mass of the heavy Higgs scalar, Haber [27] has shown that we can have

$$
\cos ^{2}(\beta-\alpha) \sim \frac{\lambda_{i} v^{2}}{m_{H^{0}}^{2}}
$$

instead of Eq. (12), while still keeping $m_{h^{0}}$ small. This can be achieved by making $m_{T}^{2} \sim m_{S}^{2}$ (see Eq. (6)), ] while keeping $m_{A^{0}}^{2} m_{L}^{2}+m_{D}^{4}<<m_{S}^{2}$ (see Eq. (5)). A simple way to arrange this is to take $\lambda_{5} \sim m_{H^{0}}^{2} / v^{2}$, while keeping all other $\lambda_{i}$ fixed. 9 Combining Eqs. (11) and (14) yields

$$
m_{H^{0}}<\left(\frac{4 \pi}{N_{c}}\right)^{1 / 2} \frac{\sqrt{\lambda_{i}} v^{2}}{m_{f}}
$$

which scales with $m_{f}$ like the Appelquist-Chanowitz bound, Eq. (11). For $\lambda_{i} \approx 4 \pi$ (the largest value of the coupling such that it is perturbative), the mass of the heavy Higgs scalar saturates this bound. This is the first example of such a model of which we are aware. However, since all the dimensionless Higgs self-couplings (including $\lambda_{5}$ ) may be restricted to be perturbative, it is not clear how to interpret this model.

Since $\cos (\beta-\alpha)$ has a lower bound given by Eq. (11), we should check that the amplitude for $V_{L} V_{L} \rightarrow V_{L} V_{L}$ is sufficiently unitarized by the light Higgs scalar $h^{0}$ that it remains within the unitarity bound up to the mass of the heavy Higgs scalar $H^{0}$. The $s$-wave, isosinglet amplitude is given by [17]

$$
a_{0}^{0}=\frac{s}{16 \pi v^{2}}\left[1-\frac{s}{s-m_{h^{0}}^{2}} \sin ^{2}(\beta-\alpha)\right] \rightarrow \frac{s}{16 \pi v^{2}} \cos ^{2}(\beta-\alpha)
$$

where the last expression is valid for $s>>m_{h^{0}}^{2}$. Using Eq. (11), this implies

$$
a_{0}^{0}>\frac{s}{16 \pi v^{2}} \frac{N_{c}}{4 \pi} \frac{m_{f}^{2}}{v^{2}}
$$

\footnotetext{
${ }^{7}$ This disrupts the cancellation mentioned in the previous footnote, such that Eq. (7) results in Eq. (14).

${ }^{8}$ This yields $s_{\beta}^{2} \sim v^{2} / m_{H^{0}}^{2}$, as follows from Eqs. (9) and (14), which is necessary to keep $m_{L}^{2}$ and $m_{D}^{2}$ small.
} 
which respects the unitarity condition $\left|\operatorname{Re} a_{0}^{0}\right|<1 / 2$ up to the energy

$$
\sqrt{s}<\frac{4 \pi \sqrt{2} v^{2}}{\sqrt{N_{c}} m_{f}} .
$$

This is essentially the Appelquist-Chanowitz bound, Eq. (11), so we are assured that the heavy Higgs scalar which unitarizes $f \bar{f} \rightarrow V_{L} V_{L}$ is also light enough to complete the unitarization of $V_{L} V_{L} \rightarrow V_{L} V_{L}$.

We have shown that in a weakly-coupled two-Higgs-doublet model, the scale of fermion mass generation is bounded by Eq. (13) rather than by the higher Appelquist-Chanowitz bound, Eq. (1). For the top quark, this bound is about $1.5 \mathrm{TeV}$, which makes it an interesting benchmark for future colliders. However, if we allow some of the dimensionless Higgs selfcouplings to become large, then we are able to saturate the Appelquist-Chanowitz bound. While this may not be physically realizable, it suggests that the Appelquist-Chanowitz bound may be relevant for strongly-coupled models of fermion mass generation. This bound is about $3 \mathrm{TeV}$ for the top quark, which is also an interesting benchmark for future colliders.

\section{Acknowledgments}

We are grateful for conversations with T. Han and J. Shigemitsu. S. W. was supported in part by the U. S. Department of Energy under contract No. DOE DE-FG02-91ER40677.

\section{References}

[1] T. Appelquist and M. Chanowitz, Phys. Rev. Lett. 59, 2405 (1987).

[2] W. Marciano, G. Valencia, and S. Willenbrock, Phys. Rev. D 40, 1725 (1989).

[3] S. Blusk, presented at the XXXIII Rencontres de Moriond on QCD and High Energy Hadronic Interactions, Les Arcs, France, March 21-28, 1998 (hep-ex/9805035).

[4] O. Éboli, G. Marques, S. Novaes, and A. Natale, Phys. Rev. D 34, 771 (1986); Phys. Lett. B178, 77 (1986).

[5] S. Dawson and S. Willenbrock, Nucl. Phys. B284, 449 (1987).

[6] C. P. Yuan, Nucl. Phys. B310, 1 (1988).

[7] F. Larios and C. P. Yuan, Phys. Rev. D 55, 7218 (1997).

[8] V. Barger, R. Phillips, and A. Stange, Phys. Rev. D 45, 1484 (1992).

[9] D. Dicus, C. Kao, and W. Repko, Phys. Rev. D 36, 1570 (1987); C. Kao and D. Dicus, Phys. Rev. D 43, 1555 (1991).

[10] E. W. N. Glover and J. van der Bij, Nucl. Phys. B321, 561 (1989). 
[11] M. Berger and M. Chanowitz, Phys. Rev. Lett. 68, 757 (1992).

[12] J.-L. Basdevant, E. Berger, D. Dicus, C. Kao, and S. Willenbrock, Phys. Lett. B313, 402 (1993).

[13] R. Kauffman, Phys. Rev. D 41, 3343 (1990).

[14] T. Barklow, in 1996 DPF/DPB Summer Study on New Directions for High-Energy Physics, Snowmass, CO, eds. D. Cassel, L. Genari, and R. Siemann (SLAC, 1997), p. 819; M. Gintner and S. Godfrey, ibid., p. 824 (hep-ph/9612342).

[15] F. Larios, T. Tait, and C. P. Yuan, Phys. Rev. D 57, 3106 (1998).

[16] Muon Quartet Collaboration (V. Barger, M. Berger, J. Gunion, and T. Han), in Future High Energy Colliders, AIP Conference Proceedings 397, Santa Barbara, Oct. 2125, 1996, ed. Z. Parsa (American Institute of Physics, Woodbury, 1997), p. 219 (hepph/9704290).

[17] M. Chanowitz and M. K. Gaillard, Nucl. Phys. B261, 379 (1985).

[18] M. Lüscher and P. Weisz, Phys. Lett. B212, 472 (1988).

[19] E. Eichten and K. Lane, Phys. Lett. B90, 125 (1980).

[20] S. Dimopoulos and L. Susskind, Nucl. Phys. B155, 237 (1979).

[21] M. Golden, Phys. Lett. B338, 295 (1994).

[22] S. Willenbrock, in Future High Energy Colliders, AIP Conference Proceedings 397, Santa Barbara, Oct. 21-25, 1996, ed. Z. Parsa (American Institute of Physics, Woodbury, 1997), p. 27 (hep-ph/9702330).

[23] R. Dashen and H. Neuberger, Phys. Rev. Lett. 50, 1897 (1983).

[24] H. Haber, G. Kane, and T. Sterling, Nucl. Phys. B161, 493 (1979).

[25] H. Georgi, Hadronic J. 1, 155 (1978).

[26] H. Haber and Y. Nir, Nucl. Phys. B335, 363 (1990).

[27] H. Haber, in Perspectives for Electroweak Interactions in $e^{+} e^{-}$Collisions, proceedings of the workshop, Ringsberg, Germany, Feb. 5-8, 1995, ed. B. Kniehl (World Scientific, Singapore, 1995), p. 219 (hep-ph/9505240); in Electroweak Symmetry Breaking, proceedings of the workshop, Budapest, Hungary, July 11-15, 1994, eds. F. Csikor and G. Pocsik (World Scientific, Singapore, 1995), p. 1 (hep-ph/9501320).

[28] M. Einhorn and G. Goldberg, Phys. Rev. Lett. 57, 2115 (1986).

[29] J. Shigemitsu, Phys. Lett. B189, 164 (1987); S. Aoki, J. Shigemitsu, and J. Sloan, Nucl. Phys. B372, 361 (1992); W. Bock, A. De, C. Frick, J. Jersák, and T. Trappenberg, Nucl. Phys. B378, 652 (1992). 
[30] M. Chanowitz, M. Furman, and I. Hinchliffe, Nucl. Phys. B153, 402 (1979). 